\title{
Über den Einfluss des fortdauernden Gebrauches von kohlensaurem Natron auf die Zusammensetzung des Blutes.
}

\author{
Von Dr. D. Dubelir aus St. Petersburg. \\ (Aus dem Laboratorium des Prof. E. Ludwig.)
}

(Vorgelegt in der Sitzung am 7. April 1881.)

Im Jahre 1843 hat Professor Nas s e ${ }^{1}$ acht Versuche ther den Einfluss des mit der Nahrung eingeführten kohlensauren Natrons auf die Zusammensetzung des Blutes mitgetheilt, die er an Hunden und Ziegen angestellt hat, nämlich an vier Hunden, die täglich 1-4 Drachmen kohlensauren Natrons mit gewöhnlicher, halb vegetabilischer, halb animalischer Nahrung bekamen, an zwei anderen Hunden, denen dabei das Kochsalz möglichst entzogen wurde, und zwei Versuche an 2 Ziegen.

In Bezug auf die äussere Beschaffenheit des Blutes fand sich: 1. meist eine hellere Farbe und ein späteres Dunkel werden; 2. eine grössere Dickflüssigkeit in den Fällen, in denen das Kochsalz vermindert; 3. eine Verlangsamung der Gerinnung, mit Ausnahme bei einem Hunde, dem das Kochsalz entzogen war; 4. verminderte Neigung der Blutkörperchen, sich mit einander zu verbinden und sich zu Boden zu senken; 5 . nur in einem Falle bẹi einem Hunde etwas trübes Serum, obgleich Bostock die trübe Beschaffenheit des Blutes nach längerem Gebrauche von kohlensaurem Natron immer gefunden haben will; 6 . eine vermehrte Fähigkeit, Kohlensäure beim Schtitteln zu absorbiren.

1 Über die arzneiliche Wirkung des kohlensauren Natrons, namentlich auf die Beschaffenheit des Blutes. Med. Correspond. Bl. rhein. Ärzte Nr. 1, 1843. Schmidts Jahrbücher 1843, Bd. XXXVIII, p. 274. 
In Bezug auf die chemischen Veränderungen des Blutes sind Folgendes die Resultate: $A$. Bei den 4 Hunden, denen nebst dem kohlensauren Alkali auch Kochsalz gegeben wurde, war 1. der Wassergehalt des Blutes im Ganzen vermehrt; 2. der des Faserstoffs etwas (um 1/, im Durchschnitte) vermindert; 3. das Fett in der normalen Menge vorhanden; 4. die Menge der löslichen Salze etwas, aber nicht regelmässig vermindert; 5. unter den einzelnen löslichen Salzen das kohlensaure, sowie das phosphorsaure Alkali in grösserer, die Chloride der Alkalimetalle und auch das schwefelsaure Alkali in geringerer Menge bemerkbar; 6 . der Gehalt an Eisen keineswegs schwächer, eher stärker, als sonst, der des Kalkes aber nicht gross. B. die Analysen des Blutes der beiden Hunde, denen das Kochsalz möglichst entzogen worden war, stimmen nur in Folgendem tiberein: 1. in einer grösseren Menge vou festen Bestandtheilen; 2 in einer Abnahme des Faserstoffes (um $1 / 5$ im Durchschnitte); 3. in Verminderung des Fettgehaltes, und 4. in einer absoluten oder relativen Zunahme des kohlensauren Alkali in Beziehung auf das phosphorsaure und schwefelsaure Salz, aber nicht auf das Kochsalz. C. In zwei an Ziegen angestellten Versuchen wurde das Blut concentrirter, d. h. die festen Bestand theile und namentlich der Faserstoff walten vor.

Auffallend ist es, dass das kohlensaure Natron in dem Blute des pflanzenfressenden Thieres gerade die entgegengesetzte Wirkung von der hervorbrachte, die im fleischfressenden Thiere beobachtet wurde. Diese Verschiedenheit mag zum Theile wenigstens von der verschiedenen Magenflitssigkeit jener Thierclassen herrühren.

Professor Nasse hat auch an Kaninchen Versuche mit dem kohlensauren Natron angestellt, und gefunden, dass, während bekanntlich bei Menschen die Verdaung geschwächt wird und Abmagerung eintritt, bei Kaninchen nicht die geringste Veränderung im Körpergewichte und in der Verdauung eintritt.

Da das kohlensaure Natron nicht bloss physiologisches Interesse hat als ein normaler Bestandtheil unseres Organismus (Lehmann, Kossel u. A.) und als Verbindung, in welche die mit der Nahrung eingenommenen Salze der Pflanzensäuren iubergehen (Wöhl er), sondern anch praktisches Interesse für den Arzt besitzt als oft grebrauchtes Medicament und als Bestandtheil vieler 
Über d. Einfluss d. Gebrauches v. kohlensaurem Natron etc.

Mineralwässer, so habe ich es unternommen, einige Versuche von Nasse za wiederholen und namentlich jene, welche folgende Fragen betreffen:

1. Ob durch längeren inneren Gebrauch von kohlensaurem Natron mit der Nahrung sich dasselbe im Blute anhäufe;

2. $\mathrm{Ob}$ und in welcher Weise sich dabei die Aschenbestandtheile des Blutes ändern;

3. welchen Veränderungen dabei der Stickstoffgehalt des Blutes unterliegt.

Diese Versuche auszuführen habe ich umsoweniger für überflüssig gehalten, als nicht alle späteren Autoren mit $\mathrm{N}$ a s s e übereinstimmen, obwohl sie ihre Schlüsse nicht immer auf Blutanalysen, sondern auf klinische Beobachtungen, auf Harnanalysen oder nur auf Reflexionen stiitzen.

So theilt Verdeil ${ }^{1}$ auf Grund seiner Blutanalysen mit, dass durch Gaben kohlensaurer Salze zwar nicht die Menge dieser im Blute, aber wohl die der mit organischen Säuren gebildeten Salze vermehrt werde.

Köhle ${ }^{2}$ sagt: „Wird das Blut durch Alkalimissbrauch in die Lage gesetzt, sich andauernd des ihm zugeführten Plus an Alkalien zu entledigen, so macht sich die antiplastische Wirkung der letzteren allmälig geltend, und eine an Scorbut erinnernde, und durch Abmagerung oder gedunsenes Aussehen, Hypostasen etc. zu erkennen gebende Kachexie (von den Franzosen Cachexie alkaline genannt) kömmt zur Entwicklung. ". . . und weiter ${ }^{3}$ :

"Das Blut stellt seinen normalen Alkaligehalt durch schnelle Elimination des Plus wieder her."

Bene k $\mathrm{e}^{4}$ gibt an, dass der andauernde Gebrauch kohlensaurer Alkalien die Ernährung herabsetze and chlorotische $\mathrm{Zu}$ stände im Gefolge habe, und erklärt es für wahrscheinlich, "dass diese chlorotischen Zustände weniger Ursache als Folge der Zunahme der alkalischen Basen im Organismuss sind."

1 F. Verdeil „Untersuchungen der Blutasche verschiedener Thiere“; Liebigs Annal. 1849, Bd. 69, 89.

2 Handbuch der phys. Therapeutik 1875, p. 94.

3 Pag. 99.

${ }^{4}$ "Grundlinien der Pathologie des Stoffwechsels" 1874, p. p. 69, 378. 
Wie Beneke geben auch Rossbach und Nothnagel ${ }^{1}$ die Möglichkeit der Anhäufung des kohlensauren Natrons zu, indem sie sagen: . . . . " erfolgt die Aufsaugung mancher Natriumsalze, wie die des schwefelsauren, kohlensauren Natriums u. s. f. wegen ihres geringen Diffusionsvermögens langsam, so' geht andererseits auch ihre Ausscheidung aus dem Blute nicht so schnell von Statten, wie z. B. die der Kaliumsalze. Die Natriumverbindungen können sich mithin leichter in grösserer Menge im Blute anhäufen."

Im Widerspruche mit den genannten Autoren steht $\mathrm{Buch}$ heìm². Er sagt nämlich Folgendes: „Der Übergang der im Darmcanale befindlichen kohlensauren Alkalien in das Blut ist hauptsächlich abhängig von dem Diffusionsvermögen derselben. Da die doppeltkohlensauren Salze nur ein geringes Diffusionsvermögen besitzen, so gehen sie nur langsam und in beschränkter Menge in das Blut über. Nach der Einführung grösserer Mengen, z. B. von kohlensaurem Natrium gelangt daher eine grössere Quantität des Salzes in den Dünndarm und wirkt hier in derselben Weise wie das Glaubersalz. Daher zeigen grössere Dosen von kohlensaurem Natrium sowie die Alkalisalze organischer Säuren, welche im Darmcanale in kohlensaure Salze ungewandelt werden, eine abfuibrende Wirkung. Dass die abführende Wirkung der doppeltkohlensauren Alkalien etwas schwächer ist, als die des Glaubersalzes, hat wohl seinen Grund in der geringeren Löslichkeit derselben. Je länger nun die doppeltkohlensauren Salze im Darmcanale verweilen, ohne mit dem Darminhalte ausgeleert zı werden, desto grössere Mengen davon können auch allmälig in das Blut uibergehen.

Wegendieses langsamen Überganges derdoppeltkohlensauren Salze kann auch, nach Einführnng grösserer Mengen davon in dem Darm keine Anhäufung derselben im Blute entstehen“" .... und weiter (p. 150) .... „Ob wir im Stande sind, durch den arzneilichen Gebrauch alkalischer Mittel einen bemerklichen Einfluss auf die Alkalescenz des Blutes auszuäben, erscheint noch

1 Handb. d. Arzneimittellehre 1880, p. 11.

2 Lehrb. der Arzneimittellehre 1878, p. 148. 
sehr zweifelhaft. Der langsame Übergang der kohlensauren Alkalien in das Blut und ihre baldige Wiederausscheidung machen eine Anhäufung derselben im Blute nicht wahrscheinlich. Eine solche wiirde auch noch sehr bald Störungen der verschiedenen Körperthätigkeiten hervorrufen, die wir beim arzneilichen Gebrauche jener Mittel nicht beobachten".

Was die zweite Frage anbelangt, so kann man die Angaben der Autoren in zwei Gruppen eintheilen: in solche, welche den Einfluss des kohlensauren Natrons auf den Säuregehalt der Blutasche, und in solche, welche den Einfluss auf den Gehalt an Basen ins Auge fassen.

So behauptet Lersch ${ }^{1}$, dass das kohlensaure Natron die Säuren im Organismus bindet, und sie durch Neutralisation oder Alkalisation (bezitglich der Phosphorsäure) fähig mache, in Salzverbindung in mehrere Säfte überzugehen und durch die Nieren auszutreten. Das Natrium entfiihrt also nach seiner Meinung dem Körper Säuren. (Chlor; Schwefelsäure, Phosphorsäure).

Nach Herrmann ${ }^{2}$ dagegen scheint der grösste Theil des kohlensauren Natrons unverändert den Organismus zu verlassen; nur ein Theil erscheint im Harne als Phosphat, wozu es jedoch keiner Umsetzung im Stoffwechsel bedarf, da im Harne selbst sich das saure Natriumphosphat mit dem Carbonat zu neutralem Phosphat umsetzen muss, ehe freies Carbonat auftreten kann:

$$
2 \mathrm{PO}_{4} \mathrm{NaH}_{2}+\mathrm{CO}_{3} \mathrm{Na}_{2}=2 \mathrm{PO}_{4} \mathrm{Na}_{2} \mathrm{H}+\mathrm{H}_{2} \mathrm{O}+\mathrm{CO}_{2} \text {. }
$$

Endlich sagt Beneke ${ }^{3}$, dass man dem Organismus durch alkalische Basen Schwefelsäure in gleicher Weise entziehen könne, wie durch schwefelsaure Alkalien.

In Beziehung auf die Basen handelt es sich hauptsächlich darum, ob tuberschüssig eingeführtes Natron im Organismus Kali vertreten kann.

1 Lersch: Die phys. u. therap. Fundam. der prakt. Balneologie und Hydrop. p. 664 .

2 Lehrb. d. experm. Toxicologie 1874, p. 187.

3 L. e. p. 363 . 
Böcker ${ }^{1}$ behauptet anf Grund seiner Untersuchungen mit phosphorsaurem Natron, dass im Organismus Kali durch Natron substituirt werden kann.

Bung $\mathrm{e}^{2}$ hat gefunden, dass die Kaliausscheidung am Tage der Natronaufnalme vermehrt wird.

Lunin ${ }^{3}$, welcher bemerkte, dass von seinen Versuchsthieren diejenigen schneller zu Grunde gingen, welche grössere Mengen von kohlensaurem Natron bekommen hatten, und erklärt dies damit, dass das Natronsalz durch Massenwirkung die anderen Salze aus dem Gewebe verdrängt hatte.

Was die dritte Frage betrifft, so ist nur eine einzige alte Beobachtung von $\mathrm{Nass} \mathrm{e}^{4}$ zu erwähnen, welcher in vier Versuchen gefunden hat, dass das kohlensaure Natron die Menge des Faserstoffes vermindert. Diese Beobachtung hat jedoch nach $\mathrm{N}$ ass e's ${ }^{5}$ eigener Angabe weit nicht den Werth, den ihr die späteren Autoren beilegen, wenn man bedenkt, mit welchen Schwierigkeiten die genaue quantitative Bestimmung des Fibrins verbunden ist. Unverständlich ist dabei die herrschende Meinung uber den Zusammenhang einer Anhäufung von Alkalien im Blute und gleichzeitige Verminderung des Faserstoffes, denn $\mathrm{N}$ ass e ${ }^{6}$ sagt ausdritcklich Folgendes:

"Besonders muss ich erinnern an das von mir entdeckte Gesetz im Thierreiche, dass Faserstoff und Alkali in einem geraden Verhältnisse zu eiṇander stehen. Auch ist das sehr gewöhnlich bei Menschen, wo der Gehalt an Faserstoff vermehrt ist, ebenso den der Alkalien vermehrt zu finden."

Zu meinem in Hinblicke auf die oben erörterten Fragen angestellten Untersuchungen diente das der Carotis entnommene Blut von Hunden, welchen während einer bestimmten Zeitdauer mit ihrer aus geniigenden Mengen von Fleisch und Brot beste-

1 Böcker: „Über die phys. Erstwirkung der Phosphors. u. d. phosphors. Natrons." Prager Vierteljahrschr. 1854, Bd. IV, p. 117.

2 B unge: „Über dieBedeutg. d. Kochsalzes u. d. Verh. d. Kalisalze im menschl. Organ. "Zeitschr. f. Biologie Bd. IX, p. 125.

3 Lunin: „Über die Bedeutg. der anorg. Salze f. d. Ernähr. d Thieres.“ Zeitschr. f. phys. Chemie v. Hoppe-Seyler V. Bd., I. Heft, 1881.

4 Wagner's Handwörterb. d. Physiologie 1842, p. 144.

5 L. e. p. 171.

6 L. c. p. 160. 
Über d. Einfluss d. Gebrauches v. kohlensaurem Natron etc.

henden Nahrung, täglich eine bestimmte Quantität von krystallisirtem kohlensaurem Natron einverleibt wurde.

Zum Vergleiche wurde auch das Blut von solchen Hunden untersucht, welche dieselbe Nahrung erhielten und unter denselben Bedingungen gehalten wurden, wie die eigentlichen Versuchsthiere, mit dem einzigen Unterschiede, dass ihrer Nahrung kein kohlensaures Natron zugesetzt war.

lch führte einerseits vollständige Analysen der Asche des Blutes aus, andererseits bestimmte ich die Menge der in dem Blute gelösten festen Bestandtheile und dessen Stickstoffgehalt. Für die Aschenanalyse $\mathrm{kam}$ die von A. Jarisch ${ }^{1}$ beschriebene Methode in Verwendung. Die Bestimmung des Stickstoffes erfolgte nach der Methode von Dumas unter Beniitzung des von Professor E. Ludwig beschriebenen Apparates ${ }^{2}$ und der von ihm angegebenen Cautelen ${ }^{3}$. Das in ganz kleinen mit gut eingeschliffenen Glasstöpseln verschliessbaren Glascylindern aufgefangene arterielle Blut wurde gewogen, in einem Tiegel gespultt, eingedampft, be $i$ $100^{\circ}$ bis $110^{\circ}$ zum constanten Gewichte gebracht und so der Trockenrickstand bestimmt. Von diesem wurde eine genau gewogene Menge mit Kupferoxyd aufs feinste zerrieben und gemengt, der Verbrennung unterzogen.

\section{A. Aschenanalysen.}

I. Normaler Hund, zwei Wochen gefiuttert mit Fleisch und Brot, 329.88 Grm. Blut lieferten $2 \cdot 8478$ Grm. $\left(0 \cdot 86^{\circ} \%\right.$ ) Asche; von dieser waren:

a) in Wasser unlöslich.... 0.3664 Grm.

b) " $"$ löslich.....2.4814 Grm.

a) der in Wasser unlösliche Theil bestand aus:

Eisenoxyd .............. 0.2300

Phosphorsäureanhydrid $\left(\mathrm{P}_{2} \mathrm{O}_{5}\right) \cdot 0 \cdot 0553$

Kalk . . . . . . . . . . . . 0.0212

Magnesia.............. $0 \cdot 0171$

$0 \cdot 3236$,

1 Wie ner medic. Jahrbüch. Jahrg. 1871 und 1877.

2 Ber. d. deutsch. chemisch. Gesellschaft in Berlin, XIII, 883.

3 Wien er med. Jahrbücher Jahrg. 1880. 
b) der in Wasser lösliche Theil bestand auș:

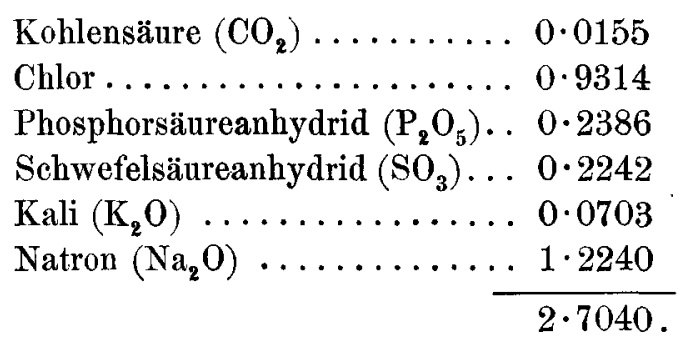

Die Metalle sind sämmtlich als Oxyde berechnet. Da aber ein Theil derselben mit dem vorhandenen Chlor als Chloride verbunden vorkommt, so muss, wenn man aus den Einzelbestinmungen die Gesammtasche berechnen will, die dem Chlor äquivalente Sauerstoffmenge von der Summe in Abzug gebrachtwerden; wir haben sodann :

Summe der in Wasser unlöslichen Bestandtheile ... 0.3236

$" \quad " \quad$ löslichen $\quad " \quad \ldots 2 \cdot 7040$ $3 \cdot 0276$ :

Für $0 \cdot 9314$ Grm. Chlor abzurechnende Sauerstoffmenge $0 \cdot 2099$ berechnete Gesammtasche . . . . . 2. 2177 direct gefundene........... $2 \cdot 8478$.

II.' Hund, zwei Wochen gefuittert mit Fleisch, Brot und 3 Grm. Soda per Tag.

$302 \cdot 85 \mathrm{Grm}$. Blut lieferten $2 \cdot 8473 \mathrm{Grm}$. $(0 \cdot 94 \%)$ Asche; von dieser waren

a) in Wasser unlöslich... 0.3404 Grm.

b) " $"$ löslich ... 2.5069 Grm. (berechnet).

a) Der im Wasser unlösliche Theil bestand aus:

Eisenoxyd . . . . . . . . . . . 0.2312 Grm.

Phosphorsäureanhydrid $\left(\mathrm{P}_{2} \mathrm{O}_{5}\right) \ldots \ldots .0 .0533$

Kalk .................. 0.0150 "

Magnesia................ $\frac{0.0165}{0.3160 .}$ 
Über d. Einfluss d. Gebrauches v. kohlensaurem Natron etc. 303

b) Dër in Wasser lösliche Theil bestand aus:

Kohlensäure $\left(\mathrm{CO}_{2}\right) \ldots \ldots \ldots \ldots . . \ldots 788$

Chlor . . . . . . . . . . . . . . . 0.8598

Phosphorsäureanhydrid $\left(\mathrm{P}_{2} \mathrm{O}_{5}\right) \ldots 0 \cdot 1811$

Schwefelsäureanhydrid $\left(\mathrm{SO}_{3}\right) \ldots .0 \cdot 2040$

Kali $\left(\mathrm{K}_{2} \mathrm{O}\right) \ldots \ldots \ldots \ldots \ldots \ldots . \ldots . \ldots 062$

Natron $\left(\mathrm{Na}_{\mathrm{z}} \mathrm{O}\right) \ldots \ldots \ldots \ldots \ldots \ldots \frac{1 \cdot 1168}{2 \cdot \frac{116}{5087}}$

Summe der in Wasser unlöslichen Bestandtheile.... $0 \cdot 3160$

$$
" \quad " \quad \text { "löslichen } \quad \ldots \frac{2 \cdot 5087 .}{2 \cdot 8247}
$$

Futr 0.8598 Chlor abzuziehender Sauerstoff . . . . . . 0 1938

berechnete Aschenmenge . . . . . . . 2.6309

direct gefundene . . . . . . . . 2 28473 .

III. Hund, sechs Wochen gefiittert mit Fleisch und Brot und 5 Grm. Soda per Tag.

$405 \cdot 87$ Grm. Blut lieferten 3.4031 Grm. $\left(0 \cdot 84^{\%}\right)$ Asche; von dieser waren:

a) in Wasser unlöslich ...... 0.4556

b) in Wasser löslich ....... 2.9475.

a) Der in Wasser unlösliche Theil bestand aus:

Eisenoxyd............ 0.3224

Phosphorsäureanhydrid $\left(\mathrm{P}_{2} \mathrm{O}_{5}\right) \ldots 0.0551$

Kalk ................ 0.0200

Magnesia............. $\frac{0.0234}{0.4209 .}$

b) Der in Wasser lösliche Theil bestand aus:

Kohlensäure $\left(\mathrm{CO}_{2}\right) \ldots \ldots \ldots . \ldots 0400$

Chlor .................. 1.1107

Phosphorsäureanhydrid $\left(\mathrm{P}_{2} \mathrm{O}_{5}\right) \ldots 0 \cdot 3046$

Schwefelsäureanhydrid $\left(\mathrm{SO}_{3}\right) \ldots 0 \cdot 2047$

Kali $\left(\mathrm{K}_{2} \mathrm{O}\right) \ldots \ldots \ldots \ldots \ldots \ldots \ldots . \ldots . \ldots .1028$

Natron $\left(\mathrm{Na}_{2} \mathrm{O}\right) \ldots \ldots \ldots \ldots \ldots \frac{1 \cdot 4992}{3 \cdot 2620}$ 
Summe der in Wasser unlöslichen Bestandtheile . . . $0 \cdot 4209$

$$
" \quad " \quad \text { "löslichen } \quad \ldots .3 \cdot 2620
$$

Für 1.1107 Grm. Chlor abzurechnender Sauerstoff. . . 0.2503 berechnete Aschenmenge. . . . . . 3.4326 direct gefundene............ 3 4031 .

IV. Hund, $30 \mathrm{Klgrm}$. Gewicht, 6 Wochen gefitttert mitFleisch und Brot und $6 \mathrm{Grm}$. Soda pr. die.

$244 \cdot 004$ Grm. Blut lieferten $2 \cdot 0523\left(0 \cdot 84^{\circ} \%\right)$ Asche; von dieser waren:

a) in Wasser unlöslich.... 0.3505 Grm.

b) " " löslich .... 1.7018 "

a) Der in Wasser unlösliche Theil bestand aus:

Eisenoxyd............... 0.2554 Grm.

Phosphorsäureanhydrid $\left(\mathrm{P}_{2} \mathrm{O}_{5}\right) \ldots \ldots .0 .0639$,

Kalk ................... 0.0150 ,

Magnesia................ 0.0138 ,

$0 \cdot 3481$.

b) Der in Wasser lösliche Theil bestand aus:

Kohlensäure $\left(\mathrm{CO}_{2}\right) \ldots \ldots \ldots, 0 \cdot 0744$

Cblor.................. 0.6128

Phosphorsäureanhydrid $\left(\mathrm{P}_{2} \mathrm{O}_{5}\right) \ldots 0 \cdot 1932$

Schwefelsäureanhydrid $\left(\mathrm{SO}_{3}\right) \ldots 0 \cdot 1298$

Kali $\left(\mathrm{K}_{2} \mathrm{O}\right) \ldots \ldots \ldots \ldots \ldots \ldots, 0.0618$

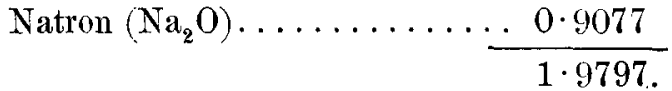

Summe der im Wasser unlöslichen Bestandtheile ... $0 \cdot 3481$

$" \quad " \quad$ " löslichen $\frac{\ldots 1 \cdot 9797}{2 \cdot 3278 .}$

Für 0.6128 Grm. Chlor abzurechnender Sauerstoff. . . $0 \cdot 1380$ berechnete Aschenmenge ....... 2.1898 direct gefundene Aschenmenge .... 2.0523. 
Ứber d. Einfluss d. Gebrauches v. kohlensaurem Natron etc.

Zusammenstellung der analytischen Resultate für 100 Theile Blutasche.

\begin{tabular}{|c|c|c|c|c|c|c|c|}
\hline & & & & & & arisch & \\
\hline & 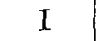 & II & III & IV & 1 & 2 & 3 \\
\hline Phosphorsäureanhy- & & & & & & & \\
\hline drid $\ldots \ldots \ldots$ & $10 \cdot 43$ & $8 \cdot 91$ & $10 \cdot 48$ & $11 \cdot 74$ & $13 \cdot 78$ & $11 \cdot 85$ & $13 \cdot 84$ \\
\hline Schwefelsäureanhy- & . & & & & & & \\
\hline drid & $7 \cdot 96$ & $7 \cdot 75$ & $5 \cdot 96$ & $5 \cdot 93$ & $4 \cdot 18$ & 4.72 & $3 \cdot 49$ \\
\hline Chlor & $33 \cdot 06$ & $32 \cdot 68$ & $32 \cdot 36$ & $27 \cdot 98$ & $31 \cdot 31$ & $33 \cdot 76$ & $33 \cdot 00$ \\
\hline Kohlensäure ...... & 0.55 & $3 \cdot 00$ & $1 \cdot 17$ & $3 \cdot 40$ & - & - & - \\
\hline Kali . . . . . . . . . & $2 \cdot 50$ & $2 \cdot 59$ & $3 \cdot 00$ & $2 \cdot 82$ & $4 \cdot 54$ & 3.54 & $3 \cdot 90$ \\
\hline Natron..... & $43 \cdot 44$ & $42 \cdot 45$ & $43 \cdot 67$ & $41 \cdot 45$ & $42 \cdot 03$ & $44 \cdot 77$ & $43 \cdot 12$ \\
\hline$\ldots \ldots \ldots$ & $0 \cdot 75$ & $0 \cdot 57$ & $0 \cdot 58$ & $0 \cdot 68$ & $1 \cdot 03$ & $1 \cdot 61$ & $1 \cdot 34$ \\
\hline Magnesia......... & $0 \cdot 60$ & $0 \cdot 68$ & $0 \cdot 68$ & $0 \cdot 63$ & $0 \cdot 81$ & $0 \cdot 75$ & 0.68 \\
\hline Eisenoxyd.... & $8 \cdot 16$ & $8 \cdot 79$ & $9 \cdot 39$ & $11 \cdot 67$ & $9 \cdot 37$ & $6 \cdot 58$ & $8 \cdot 06$ \\
\hline & $107 \cdot 45$ & $107 \cdot 37$ & $107 \cdot 29$ & $106 \cdot 30$ & $107 \cdot 05$ & $107 \cdot 58$ & $107 \cdot 43$ \\
\hline $\begin{array}{l}\text { Für Chlor abzuzie- } \\
\text { hender Sauerstoff }\end{array}$ & $7 \cdot 45$ & $7 \cdot 37$ & $7 \cdot 29$ & $6 \cdot 30$ & $7 \cdot 05$ & $7 \cdot 58$ & $7 \cdot 43$ \\
\hline Summe & $100 \cdot 00$ & 100.00 & $100 \cdot 00$ & $100 \cdot 00$ & $100 \cdot 00$ & $100 \cdot 00$ & $100 \cdot 00$ \\
\hline
\end{tabular}

In 100 Theilen Blut sind enthalten:

\begin{tabular}{|c|c|c|c|c|c|c|c|}
\hline & \multirow[b]{2}{*}{$\mathrm{T}$} & \multirow[b]{2}{*}{ II } & \multirow[b]{2}{*}{ III } & \multirow[b]{2}{*}{ IV } & \multicolumn{3}{|c|}{ Jarisch } \\
\hline & & & & & 1 & 2 & 3 \\
\hline $\begin{array}{l}\text { Posphorsäureanhy- } \\
\text { drid .......... }\end{array}$ & 0.0861 & $10 \cdot 0774$ & 0.0886 & $0 \cdot 1054$ & $\mid 0 \cdot 1191$ & 0.1062 & $0 \cdot 1193$ \\
\hline $\begin{array}{l}\text { Schwefelsäureanhy- } \\
\text { drid .......... }\end{array}$ & 0.0680 & 0.0674 & 0.0504 & 0.0532 & 0.0362 & 0.04234 & 0.0298 \\
\hline Chlor ......... & $0 \cdot 2823$ & $0 \cdot 2839$ & $0 \cdot 2737$ & $0 \cdot 2511$ & $\mid 0 \cdot 2705$ & $0 \cdot 3026]$ & 0.2821 \\
\hline Kohlensäure....... & $0 \cdot 0047$ & $|0 \cdot 0260|$ & $0 \cdot 0099$ & 0.0305 & - & - & - \\
\hline Kali ............. & $0 \cdot 0213$ & 0.0225 & $0 \cdot 0253$ & $0 \cdot 0253$ & $0 \cdot 0392$ & $0 \cdot 0318$ & $0 \cdot 0333$ \\
\hline Natron.... & 0.3710 & $0 \cdot 3688$ & $0 \cdot 3694$ & $0 \cdot 3717$ & $0 \cdot 3631$ & $|0 \cdot 4012|$ & $0 \cdot 368$ \\
\hline Kalk ......... & $0 \cdot 0064$ & $0 \cdot 0050$ & $0 \cdot 0049$ & 0.0061 & $0 \cdot 0090$ & 0.014 & 0.0114 \\
\hline Magnesia........ & $0 \cdot 0052$ & $0 \cdot 0054$ & $0 \cdot 0058$ & $0 \cdot 0057$ & $0 \cdot 0070$ & $0.0067 \mid$ & 0.0058 \\
\hline Eisenoxyd... & $0 \cdot 0697$ & $0 \cdot 0763 \mid$ & $0 \cdot 0794$ & $0 \cdot 1047$ & $0 \cdot 0809$ & $0 \cdot 1412$ & $0 \cdot 0688$ \\
\hline Gesammtasche gef. & 0.8633 & $0 \cdot 9402$ & $0 \cdot 8385$ & $0 \cdot 8+11$ & $0 \cdot 8856$ & $0 \cdot 8971$ & 0.9106 \\
\hline ber. . & $0 \cdot 8541$ & $0 \cdot 8687$ & $0 \cdot 8455$ & $0 \cdot 8758$ & $0 \cdot 86 \pm 3$ & $0 \cdot 8969$ & $0 \cdot 8562$ \\
\hline
\end{tabular}

1 Wien. Med. Jahrbücher Jahrg. 1877, H. I., p. 59. 
$\mathrm{Ob}$ sich die alkalisehe Beschaffenheit des Blutes jener Thiere, die mit ihrer Nahrung kohlensaures Natron aufnahmen, quantitativ geändert hat, wird man am besten beurtheilen können, wenn man die Summe der Säureäquivalente (mit Ausschluss der Kohlensäure) mit der Summe der Basenäquivalente des löslichen Theiles der Blutasche vergleicht; die folgende Tabelle enthält eine diesbezügliche Zusammenstellung, welche sowohl meine als auch die von J aris ch ${ }^{1}$ ausgeführten Analysen umfasst. Die Rechnungen für diese Tabelle sind unter der von $B$ unsen ${ }^{2}$ gegebenen Voraussetzung angestellt, dass die Phosphorsäure als Salz von der Form $\mathrm{Na}_{2} \mathrm{HPO}_{4}$ in dem löslichen Theile der Asche erscheint.

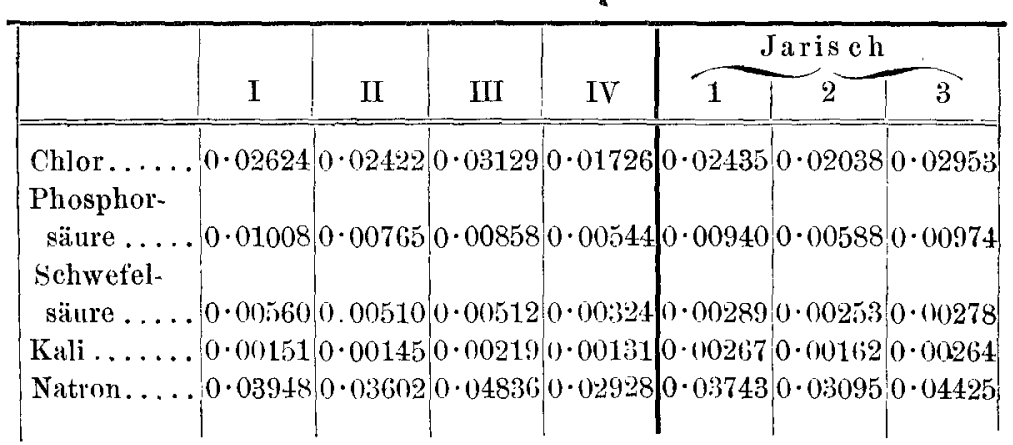

Nach dieser Tabelle gestaltet sich das Verhältniss zwischen den Summen der Sätre- und Basenäquivalente, wie folgt:

\begin{tabular}{|c|c|c|c|c|c|c|c|}
\hline & & & & & & Talise & \\
\hline & $\mathrm{I}$ & II & III & IV & 1 & 2 & 3 \\
\hline Säureäquivalent & 1 & 1 & 1 & 1 & 1 & 1 & 1 \\
\hline Basenäquivalent & $1 \cdot 063$ & $1 \cdot 089$ & $1 \cdot 124$ & $1 \cdot 175$ & $1 \cdot 094$ & $1 \cdot 131$ & $1 \cdot 115$ \\
\hline
\end{tabular}

\section{B. Stickstoffbestimmungen.}

5. Normaler Hund von $25 \mathrm{Klgrm}$. Gewicht; gefittert mit Brot und Fleisch durch zwei Wochen.

1 L. c.

2 Anleitung zur Analyse der Asehen und Mineralwasser 1874, pg. 14. 
Über d. Einfluss d, Gebrauches v. kohlensaurem Natron etc. 307

6.1874 Grm. Blut gaben $1 \cdot 2317$ Grm. (19.9\%) festen Riuckstand. Davon wurden $0 \cdot 2585 \mathrm{Grm}$. zur Stickstoffbestimmung verwendet und gaben folgende Zahlen:

$$
\begin{gathered}
V=31.75 \mathrm{CC} . \\
t=8.8^{\circ} \mathrm{C} . \\
b=755 \mathrm{Mm} .
\end{gathered} \quad \begin{aligned}
\text { entspricht reducirt auf } 0^{\circ} \text { und } 760 \mathrm{Mm} .: \\
27 \cdot 81 \mathrm{CC} . \mathrm{N} .
\end{aligned}
$$

6. Normaler Hund von $4 \mathrm{Klgrm}$. Gewicht, zwei Wochen gefiuttert mit Brot und Fleisch.

$3 \cdot 6419$ Grm. Blut gaben $0 \cdot 6737$ Grm. $(18 \cdot 5 \%$ festen Rückstand. Davon gaben $0 \cdot 2026 \mathrm{Grm}$ :

$$
\left.\begin{array}{rl}
V & =26 \cdot 5 \mathrm{CC} . \\
t & =14 \cdot 2^{\circ} \mathrm{C} . \\
b & =738 \mathrm{Mm} .
\end{array}\right\} \text { reducirt auf } 0^{\circ} \text { und } 760 \mathrm{Mm} .: 24 \cdot 12 \mathrm{CC} . \mathrm{N} .
$$

7. Für diese Bestimmung wurde das Blut des Hundes IV verwendet.

$6 \cdot 2666$ Grm. Blut gaben $1 \cdot 5163(24 \cdot 2 \%)$ festen Rückstand. Davon gaben $0 \cdot 4605$ Grm.:

$$
\left.\begin{array}{rl}
\boldsymbol{V} & =63 \cdot 8 \mathrm{CC} . \\
t & =12 \cdot 0^{\circ} \mathrm{C} . \\
\boldsymbol{b} & =747.5 \mathrm{Mm} .
\end{array}\right) \text { redueirt auf } 0^{\circ} \text { und } 760 \mathrm{Mm} .: 59 \cdot 3 \quad \mathrm{CC} . \mathrm{N} .
$$

8. Kleiner Hund, 2 Klgrm. Gewicht, noch jung; gefüttert mit Fleisch und Brot und $0.5 \mathrm{Grm}$. Soda täglich.

$6 \cdot 1473$ Grm. Blut gaben $1 \cdot 0924(17 \cdot 8 \%$ ) festen Rüekstand. Davon $0 \cdot 4144 \mathrm{Grm}$. verwendet:

$$
\left.\begin{array}{rl}
V & =55.6 \mathrm{CC} . \\
t & =13 \cdot 0^{\circ} \mathrm{C} . \\
b & =742.5 \mathrm{Mm} .
\end{array}\right\} \text { reducirt auf } 0^{\circ} \text { and } 760 \mathrm{Mm} .: 51 \cdot 07 \mathrm{CC} . \mathrm{N} .
$$

Die folgende Tabelle enthält die in den vier Versuchen gefundenen Werthe fiir den Gehalt an festen Bestandtheilen, sowie an Stickstoff (Eiweisskörpern) auf 100 Theile Blut berechnet; den von mir gefundenen Werthen habe ich noch die von Forster ${ }^{1}$ beigefügt, welche sich auf einen mit vollständiger Nahrung gefütterten Hund beziehen.

1 Zeitschr. f. Biolog. Bd. IX, p. 363. 
Feste Bestand-

$$
\text { theile } \mathrm{N} \text {. }
$$

Vollständige Nahrung......... 22.2 $3 \cdot 22$ (Forster)

Normaler Hund . . . . . . . . . . . . 19.9 2.69

Normaler Hund ............ 18.5 $2 \cdot 77$

Nach 6wöchentlichem Gebrauche von

$6 \mathrm{Grm}$. Soda täglich.......... 24.2 $3 \cdot 90$

Nach 3wöchentlichem Gebrauche von

$0.5 \mathrm{Grm}$. Soda täglich ........ $17 \cdot 8 \quad 2 \cdot 75$.

Auf Grund dieser allerdings geringen Zahl von Analysen lassen sich folgende Schlüsse ziehen (zunächst für den Organis-. mus des Hundes):

1. Die alkalische Beschaffenheit der Blutasche erfährt bei fortdauerndem Gebrauche grösserer Gaben von Soda eine kleine, immerhin aber merkliche Vergrösserung, die mit der täglichen Menge der eingeführten Soda und der Zeitdaner, während welcher sie eingeführt wird, wächst.

2. Kali wird in der Blutasche nicht dureh Natron substituirt.

3. Natron wird im Blute nicht angehäuft.

4. Der Eisengehalt wird, wie schon Nasse bemerkt, nicht vermindert.

5. Der Gehalt des Blutes an festen Bestandtheilen, sowie an Stickstoff (Eiweiss) wird durch den innerlichen Gebrauch von Soda nicht in solchem Grade verändert, dass er die normalen Grenzen tiberschritte, welche letzteren allerdings sehr schwankend gefunden wurden.

So fand Collard de Martigny .... 17.6\% ${ }^{1}$

$" \quad$ Forster........... $22 \cdot 2 \%$.

1 Nasse 1. c. 